\title{
Le langage de la révélation en phénoménologie radicale
}

\section{Giuliano Sansonetti (Université de Ferrare)}

\begin{abstract}
La vie est l'apparaître originel que nous désignons désormais sous le titre de révélation. [...] Le premier trait de la révélation de la vie, c'est qu'elle s'accomplit comme une autorévélation. S'autorévéler pour la vie veut dire: s'éprouver soi-même [...] ; la façon dont la vie révèle, c'est celle dont elle parle [...]; la parole que parle la vie présente le caractère extraordinaire qu'elle ne parle jamais que de soi. ${ }^{1}$
\end{abstract}

Cet extrait de «Phénoménologie matérielle et langage », un des textes de Michel Henry qui clôt les actes du colloque de Cerisy, résume parfaitement l'aboutissement de sa pensée, caractérisé par l'étroite connexion entre révélation et langage, soit entre le thème fondamental de son commencement — on sait que son opus magnum devait s'intituler L'essence de la révélation - et celui du langage, qui constitue comme la dominante de sa dernière pensée. En effet, si dans L'essence de la manifestation, le langage ne se trouve saisi que de manière latente et ne fait son apparition explicite, à propos du sentiment, qu'au $\S 61$, on peut dire qu'à partir du texte que nous avons cité, il devient un vrai leitmotiv de la pensée henryenne, qui se conclue justement sur des paroles, les paroles du Christ, paroles par excellence. C'est que, comme chez tout grand penseur, ce qui vient à la fin est en réalité présent dès le début, de sorte que ce qui n'avait d'abord qu'une présence furtive se révèle in fine obéir à une nécessité interne. Cela n'annule évidemment pas l'effectivité du chemin, au terme duquel le langage est devenu non seulement un passage obligé, mais aussi un point d'arrivée, voire une sorte de banc d'essai où se joue la validité de la phénoménologie matérielle ou radicale. Cet aboutissement, on peut le résumer dans cette affirmation : pas de révélation sans langage, pas de langage sans révélation. Toutefois, avant Michel Henry, c'est bien ce qu'avait on ne peut mieux dit Heidegger. De quelle révélation s'agit-il dès lors, et de quel langage ? Plus encore : de quel langage pour quelle révélation? Voilà la question. C'est sur ce plan que la pensée de Michel Henry croise inévitablement la pensée de Heidegger qui, dès les premières lignes de L'essence de la manifestation, se présente comme son interlocuteur privi-

\footnotetext{
${ }^{1}$ M. Henry, «Phénoménologie matérielle et langage (ou pathos et langage) », dans A. David et J. Greisch (eds.), Michel Henry, l'épreuve de la vie, Paris, Cerf, 2001, p. 23-25, repris dans Phénoménologie de la vie, tome III. De l'art et du politique, Paris, PUF, « Epiméthée », 2004, p. 334-335.
} 
légié, voire son adversaire attitré. Et c'est justement dans la connexion entre révélation et langage que se décidera à la fois la proximité et la distance majeure entre les deux penseurs, liées à l'alternative que constituent leurs deux projets d'ontologie phénoménologique.

Si l'incipit de L'essence de la manifestation répète la fameuse objection de Heidegger au cogito sum de Descartes, ce n'est pas parce que Henry en reconnaît le bien fondé, mais uniquement à cause d'un enjeu ontologique qu'il fait totalement sien: «Le sens de l'être de l'ego est le thème des présente recherches » — de sorte que c'est l'ego, et non le Dasein, l'être-au-monde, qui constituera le fil conducteur de la démarche. Cela implique que l'évidence, la vérité, qui pour Descartes réside dans l'immédiateté de la certitude, doit pouvoir se montrer de soi-même, sans qu'on doive recourir à la détermination du sens de l'être en général — sans qu'on doive, pour le dire avec Heidegger, " poser la question de savoir dans quelle mesure l'être du sujet doit être déterminé comme point de départ de la problématique philosophique $\gg .{ }^{1}$ En renversant de fond en comble la démarche heideggérienne, Henry affirme alors que "c'est seulement à partir d'une élucidation du phénomène central de l'ego que l'ontologie peut acquérir sa dimension fondamentale ». ${ }^{2}$ Contre toute une tradition de pensée qui, de Kant, va jusqu'à Heidegger, et pour laquelle aucune connaissance de l'ego ne peut faire abstraction de la connaissance du monde, le moi devient donc l'objet d'une «connaissance absolue » qui n'est pas assujettie aux conditions transcendantales de la connaissance du monde. Contre la critique heideggérienne de la pensée moderne comme «métaphysique de la subjectivité », Henry poursuit par conséquent une interprétation positive de la démarche cartésienne fondée sur l'autoévidence de l'ego hors de tout contexte mondain, et sur son identification avec le fondement de la vérité. L'ego s'affirme ainsi comme la révélation première et fondamentale, comme l'essence même de la manifestation : "L'être phénoménologique de l'ego est un avec la révélation originaire qui s'accomplit dans une sphère d'immanence radicale $\rangle^{3}$ Par là même, avec une précision absolue, se trouve déterminé le statut de la révélation qui, malgré 1'attitude radicalement critique entretenue à son égard, signe bien l'appartenance de Michel Henry au mouvement phénoménologique. Parler en effet de révélation à propos d'une immanence radicale implique la possibilité d'une phénoménologie de l'invisible en tant que tel, c'est-à-dire implique de penser une manifestation qui n'advient pas par le biais de la visibilité. L'immanence ne doit donc pas être conçue comme une sorte de résidu phénoménologique, comme ce qui se contenterait de résister au travail de la réduction, mais bien plutôt comme un «quelque chose » d'essentiel en mesure de manifester ses articulations propres et sa structure. Nous sommes ainsi renvoyés d'une

\footnotetext{
${ }_{1}^{1}$ M. Heidegger, Problèmes fondamentaux de la phénoménologie, Paris, Gallimard, 1985, p. 192.

${ }^{2}$ M. Henry, L'essence de la manifestation, Paris, PUF, « Epiméthée », 1990, p. 46.

${ }^{3}$ Ibid., p. 52.
} 
détermination critique et négative de l'essence à sa détermination positive comme pathos et affectivité, et à sa définition comme Vie : «Ce qui est impliqué dans cette positivité comme la constituant, c'est la relation de l'essence avec soi, relation telle qu'en elle l'essence jouit de soi, a l'expérience de soi, se révèle à elle-même dans ce qu'elle est, telle qu'elle est ». ${ }^{1} \mathrm{~L}$ 'essence de la manifestation n'est donc rien d'autre que la vie, visée dans son autosuffisance et son immanence. C'est pourquoi «la Parousie est l'essence de la vie $»^{2}$ : la vie réside justement dans son « se révéler», son « se recueillir » et son « coïncider avec soi ».

À la notion de vérité comme vision, comme theoria, comme ce qui se montre dans un horizon de visibilité, s'opposent donc une autre vérité et une autre manifestation qui baignent dans l'essence même de la vie en son immanence radicale. Car la vie est justement ce qui n'apparaît pas et pourtant se manifeste, dans la seule manière qu'elle a de se manifester, celle du pathos, de l'affectivité en laquelle réside son essence même. L'invisible devient ainsi l'objet d'une « révélation positive et fondamentale ». Henry n'entend pas l'immanence comme ce qui est requis par la transcendance pour s'expliquer, comme ce qui diffère de celle-ci par le manque d'horizon et de visibilité, car elle s'affirme en soi — en sa Selbständigkeit, dit-il à la suite de Heidegger — , bref, dans la pleine positivité de son être et de sa révélation. La critique du monisme ontologique ne peut en effet revendiquer sa légitimité qu'à la seule condition de montrer que la manifestation ne se recouvre pas avec la visibilité du monde, qu'il y a une autre manifestation, celle de l'essence comme immanence : «L'opposition du visible et de l'invisible, loin d'impliquer leur insertion dans l'unité dialectique d'un seul processus, exprime au contraire, comme opposition du réel et de l'irréel, l'hétérogénéité ontologique radicale de leurs essences et trouve en elle son fondement $\gg{ }^{3}$

Quel est donc le rapport entre le visible et l'invisible, l'immanence et la transcendance? Qu'il ne soit pas dialectique signifie que les termes ne s'impliquent pas, ni par opposition, ni par indifférence à la manière de Schelling. L'invisible ne doit pas être visé comme le degré zéro de ce qui se montre, ce qui reviendrait à l'entendre par défaut à partir du visible, non dans la positivité de son essence, de sa Selbständigkeit : le visible et l'invisible « subsistent l'un à l'écart de l'autre, chacun dans la positivité de son effectivité propre ». ${ }^{4}$ L'invisible n'est également « rien qui soit audelà $d u$ visible, rien de "transcendant", il est l'essence originale de la vie telle que, s'accomplissant dans une sphère d'immanence radicale, elle ne se lève jamais dans la transcendance et ne peut non plus se montrer en elle ». ${ }^{5}$ Que l'invisible ne se

\footnotetext{
${ }^{1}$ Ibid., p. 354.

${ }^{2}$ Ibid., p. 355.

${ }^{3}$ Ibid., p. 564.

${ }^{4}$ Ibid., p. 561.

${ }^{5}$ Ibid., p. 568.
} 
résolve pas dans le visible implique qu'il se révèle de façon tout à fait propre, comme affectivité.

Encore une fois, nous sommes renvoyés au rapport de Henry avec la pensée de Heidegger, en particulier au rôle de la Befindlichkeit dans l'analytique existentiale d'Être et temps, où elle se trouve conçue comme tonalité affective du comprendre (Verstehen). Il s'ensuit que si chez Heidegger le pouvoir de l'affectivité consiste uniquement dans la compréhension ontologique de l'être, sans laquelle il n'y aurait pas compréhension du Dasein, c'est à la condition d'attribuer un pouvoir originairement « révélateur » à l'affectivité. Bien plus, celle-ci constitue la racine même de la compréhension car, comme l'écrit très justement Henry, «l'affectivité a déjà accompli son œuvre quand se lève le monde ». ${ }^{1} \mathrm{C}$ 'est que, tandis que chez Heidegger l'être est quelque chose d'énigmatique, qui s'enfouit et se retire dans le moment même où il nous affecte, chez Henry, l'invisible de l'être se recouvre avec son « être affection », avec sa révélation dans l'affectivité et comme affectivité. Celle-ci doit donc être reconnue comme le fondement même de la manifestation, et non plus seulement comme la tonalité affective qui l'accompagnerait, et ainsi comme le fondement des phénomènes dans la mesure où ceux-ci sont affectifs dans leur essence même. La conséquence est tout à fait évidente : "Ce n'est pas ce qui arrive qui détermine l'affectivité mais l'affectivité rend possible la venue de ce qui vient et le détermine $[. .$.$] comme affectif \gg{ }^{2}$

Une fois posée l'affectivité comme mode propre de manifestation, radicalement différent de la phénoménalité comme visibilité et transcendance, il faut l'entendre en toute sa transparence, comme révélation de fond en comble, et non, au risque de la falsifier complètement, comme quelque chose d'opaque et d'obscur dont le principe serait externe à l'affectivité même. Dans l'affectivité il faut distinguer deux aspects : le «contenu » et le « comment». Le «comment», l'aspect vraiment décisif pour Henry, se résume dans l'affirmation selon laquelle "l'affectivité révèle comme affectivité $\gg{ }^{3}$; en d'autres termes, ce n'est pas par la connaissance que nous pouvons la viser. Partant, quand on parle de l'obscurité de la vie affective, c'est parce qu'il s'agit d'un domaine qui se soustrait radicalement au regard, hétérogène à son égard, si bien que "l'obscurité de l'invisible ouvre la dimension ontologique où le sentiment trouve son existence originelle ${ }^{4}{ }^{4}$

En ce sens, la pensée va immédiatement à «ce Fond qui est Abîme » de Meister Eckhart, cité par Henry, «Fond absolument simple » qui laisse hors de soi toute détermination, toute différence. Fond abyssal, qui pourtant n'est pas un fond obscur, cet abîme sans lumière propre à la vision romantique de l'Absolu, résolument refu-

\footnotetext{
${ }^{1}$ Ibid., p. 604.

${ }^{2}$ Ibid., p. 611.

${ }^{3}$ Ibid., p. 674.

${ }^{4}$ Ibid., p. 680.
} 
sée par Henry. Ce Fond est plutôt révélation et, comme le Logos du Prologue de Jean, manifestation de Dieu. En effet, chez Henry, un lien direct relie le Fond eckhartien à ce Logos johannique que C'est moi la vérité déploiera comme révélation et comme Vie. Que l'essence immanente de la vie, son invisibilité, forme le contenu de la prédication du Christ, en opposition à la vérité du monde, c'est ce que Henry affirme dès L'essence de la manifestation - ce qui revient à dire que le dualisme christianisme/monde est ontologique plus encore qu'éthique. Jean-Louis Chrétien a donc raison d'affirmer que « la philosophie de Michel Henry n'aboutit pas au Logos qu'il appelle johannique (si ce n'est dans la chronologie de ses œuvres), elle en provient ». ${ }^{1}$ Plus discutable est l'autre affirmation, selon laquelle « la trilogie christologique [...] donne une intelligibilité plénière à des thèses philosophiques restées obscures ou fragiles en certains de leurs aspects $\gg .^{2}$ Je pense au contraire que c'est justement la phénoménologie de l'invisible élaborée dès les premiers ouvrages qui fait la force et la cohérence de celle qui se présente - il ne faut pas l'oublier comme une interprétation philosophique du christianisme. C'est en effet ce même Logos qui, à la lumière de la méditation des textes chrétiens, sera dit johannique, qu'on trouve déjà à l'œuvre dans L'essence de la manifestation comme langage de l'affectivité, de la vie comme affectivité. C'est ainsi que le thème du langage vient croiser celui de la révélation.

Quel rapport entretient la révélation, le fait qu'elle se produise dans l'invisible, avec le langage, ou le système des signes et des renvois qui constitue l'ordre du monde ? Il faut le répéter : si l'essence de la manifestation est la manifestation de l'essence, et si l'essence est la vie, alors la manifestation est manifestation de la vie, son autorévélation. Mais comment parle la vie ? Quelle est sa parole ? Henry écrit :

L'affectivité telle qu'elle se révèle originairement en elle-même et surgit dans la force de sa présentation phénoménologique spécifique, comme affective et comme ce qu'elle est, est l'essence originelle du Logos, de telle manière que celui-ci refuse le langage du monde, le langage de la pensée et ne peut se montrer en lui. Mais le langage est l'être. Qu'il réside originellement dans l'affectivité interdit de comprendre celui-ci comme il le fut depuis Parménide jusqu'à nos jours, à partir de la pensée et comme lui étant identique. ${ }^{3}$

Ainsi, le Logos en question ne fait pas un avec le langage de la pensée — de l'être identique à la pensée - ce langage qui, comme Heidegger l'a bien montré, a dans le leghein son essence propre. Écoutons le penseur allemand: «Dire, c'est leghein. Bien considérée, cette phrase [...] donne un nom à ce secret impensable : le parler

\footnotetext{
${ }^{1}$ J.-L. Chrétien, "Vie et immanence. La parole selon Michel Henry », dans J.-M. Brohm et J. Leclercq (eds.), Michel Henry, L'Age d'Homme, « Dossier H », Lausanne, 2009, p. 159.

${ }^{2}$ Ibid., p. 158-159

${ }^{3}$ M. Henry, L'essence de la manifestation, op. cit., p. 688-689.
} 
du langage se produit à partir de la non-occultation des choses présentes et se détermine comme le laisser-étendu-ensemble-devant, conformément au fait que la chose présente est étendue devant nous ». ${ }^{1}$ Le langage parle ainsi à partir d'un dévoilement originaire; il est moins un principe de révélation que son effet. Ce qu'exprime l'importante affirmation : "si tant est que l'essence de la parole ne peut pas ressortir de l'ordre de la parole (das Wesen der Sprache nichts Sprachliches sein kann)», alors « la parole directrice, la voilà : L'essence de la parole : La parole de l'essence (Wesens) ${ }^{2}{ }^{2}$ Or c'est ce que Henry ne peut que faire sien, au point de déclarer : Je suis redevable à Heidegger, que j'ai quitté, que j'ai pas mal critiqué, d'une des ses grandes intuitions, que je trouve admirable, et c'est justement sa conception du langage. Pourquoi ? Parce que c'est une conception phénoménologique qui présuppose ou qui comprend que le langage repose sur l'apparaître ou, comme nous disons, sur la phénoménalité pure... ${ }^{3}$

Ce avec quoi, au contraire, le désaccord ne peut être que net et radical, c'est avec la vision du langage, du leghein, comme «montrer, laisser apparaître, présenter un monde dans une éclaircie qui est du même coup abritement, les deux s'unissant en libre-donation » (Gegen-einander-über). ${ }^{4}$ En affirmant que le langage à été visé dès Parménide jusqu'à nos jours « à partir de la pensée et comme lui étant identique », Henry ne fait pas d'exception pour Heidegger - ce qui, du reste, est tout à fait cohérent avec sa critique de la Befindlichkeit réduite à une compréhension. Bref, ce que Heidegger pense sous le syntagme de « dire originaire », qui trouve son expression majeure dans le langage poétique, comme le montre en particulier l'analyse du poème de Trakl, finit lui aussi par s'inscrire dans le langage du monde, « reposant sur l'apparaître du monde $»^{5}$

À cette conception du langage, dans la droite ligne de la révélation comme apparaître, comme «dimensionnalité extatique », Henry oppose une vision qui se soustrait radicalement au paradigme de la visibilité, au nom de la «phénoménalité pure », de l'affectivité comme affectivité. Ainsi peut d'ailleurs seule être comprise l'affirmation heideggérienne selon laquelle nous écoutons la parole car nous lui appartenons (Wir hören Sie nur weil wir in Sie gehören). ${ }^{6}$ Comment définir, en effet, une telle appartenance? Est-elle définie uniquement par le comprendre, ou bien le

\footnotetext{
${ }^{1}$ M. Heidegger, Essais et conférences, Paris, Gallimard, 1958, p. 257.

${ }^{2}$ M. Heidegger, Acheminement vers la parole, Paris, Gallimard, 1976, p. 109 et p. 185 ; traduction modifiée.

${ }^{3}$ Phénoménologie de la vie, tome IV. Sur l'éthique et la religion, Paris, PUF, « Epiméthée », 2004, p. 238.

${ }^{4}$ M. Heidegger, Acheminement vers la parole, op. cit., p. 200-201

5 «Débat autour de l'œuvre de Michel Henry », dans Phénoménologie de la vie, tome IV, op. cit., p. 238.

${ }^{6}$ Cité par M. Henry dans « Parole et religion : la parole de Dieu », ibid., p. 195.
} 
comprendre repose-t-il lui-même sur quelque chose de plus originaire, telle la vie dans son autoaffection?

La réponse de la phénoménologie matérielle n'est pas douteuse, et ce n'est pas un hasard si la redéfinition radicale de la nature du langage par Michel Henry s'opère, avant C'est moi la vérité, dans Phénoménologie matérielle - lieu d'une mise au point par le philosophe de la distance qui le sépare de la phénoménologie husserlienne, et en particulier du « virage thématique » dont elle serait responsable. On sait que l'objection majeure de Henry à la phénoménologie husserlienne est que, tout en reconnaissant un rôle décisif à l'affectivité, elle finit par la dévaluer pour autant qu'elle ne la vise pas en elle-même mais uniquement en tant qu'elle participe de l'essence générale de la conscience comme conscience intentionnelle. S'accomplit ainsi subrepticement une transposition de la sphère affective sur un plan qui n'est plus affectif. Et cela parce que, comme le dit Husserl, «l'hylétique pure se subordonne à la phénoménologie de la conscience transcendantale » (Ideen I, § 86). La phénoménologie abandonne alors le domaine de l'autodonation originaire pour celui de la donation intentionnelle, tout à fait secondaire et dérivée. De cette manière, la vie se trouve transposée sur un plan qui est celui de la représentation, à savoir de la re-présentation, qui n'est plus celui de la présentation originaire, de l'Ur-phänomen. Il s'ensuit que les contenus hylétiques, matériels, dans leur immanence pure, ne deviennent lisibles qu'à la seule condition de leur transformation en noèmes conscientiels par l'intentionnalité. Et, de la même manière, le vécu n'acquiert dès lors de réalité qu'au moyen de la représentation, et au détriment de l'immédiation pathétique qui le définit comme réel. Comme l'écrit Henry, « les choses diffèrent totalement selon qu'elles sont immergées dans le pathos de la vie [...] ou qu'elles se tiennent au contraire devant un regard $\gg{ }^{1} \mathrm{C}$ 'est donc le grand thème de la donation qui est ici en question. Comme on sait, chez Husserl, la conscience intentionnelle est seule donatrice de sens, source de toute Sinngebung. Mais comme le relève Henry, la phénoménologie husserlienne mobilise en réalité une double donation : l'une, énigmatique, s'accomplit comme Empfindung, comme donné impressionnel totalement immanent ; c'est celui-ci qui constitue le moment authentiquement transcendantal de la donation. Mais pour que cette donation soit effective, une autre donation est requise qui, par l'intermédiaire de l'intentionnalité, transforme le donné impressionnel, hylétique en quelque chose de «transcendant et irréel», en son «vis-àvis ». ${ }^{2}$ Et c'est justement cette deuxième donation qui retient l'attention de Husserl. De cette manière, la phénoménologie finit elle aussi par s'inscrire dans l'horizon du monisme ontologique qui conçoit l'essence du Logos comme "phénoménalité du monde », en procédant en même temps à l'évacuation de l'affectivité de la vie, qui est la vie. D'où, selon Henry, la nécessité d'un renversement total du principe fondateur de la phénoménologie, que l'on peut résumer ainsi : "loin que ce soit la vue

\footnotetext{
${ }^{1}$ M. Henry, Phénoménologie matérielle, Paris, PUF, « Epiméthée », 1990, p. 11.

${ }^{2}$ Ibid., p. 26.
} 
pure qui nous fasse connaître sous l'aspect de son essence noématique la réalité absente ou perdue de la vie, c'est la révélation immanente de celle-ci dans la cogitatio qui donne au voir tout ce qu'il peut voir et savoir d'elle, et pour autant qu'il le puisse $»{ }^{1}$

C'est à la lumière de ce renversement qu'il faut repenser l'essence du langage, comme il advient déjà dans Phénoménologie matérielle où, pour la première fois, le Logos de l'évangile de Jean est interprété moins comme Verbe que comme Vie. Comme l'écrit Henry, « le Verbe qui vient en ce monde n'est pas le logos grec, la venue au monde elle-même en tant que telle. Ce qui vient dans le monde s'y dérobe bien plutôt, est la vie cachée ». ${ }^{2}$ Alors le propre du langage n'est plus de faire voir, en tous cas pas dans le sens de la transcendance du monde, de l'ostension dans le dehors ; bien plutôt doit-il donner la parole à la vie cachée, à l'immanence, car la parole est avant tout parole de la vie, par laquelle la vie se manifeste en soi, en ce qu'elle est. C'est la parole avant la parole dont parle C'est moi la vérité ou, comme le disent ces pages de Phénoménologie matérielle que nous commentons, c'est le dire originaire comme "plénitude phénoménologique de la vie en sa positivité infrangible », et ceci contre un langage assimilé à un meurtre, " au meurtre de la réalité supprimée et conservée sous la forme idéale du mot » ${ }^{3}$ (Henry faisant ici, de toute évidence, référence à Lacan et à la conception du langage qui, via Kojève, remonte à Hegel). D'où l'affirmation d'une duplicité du langage, que la méditation de la vérité du christianisme ne fera que confirmer. En découle alors la distinction fondamentale entre « vérité du christianisme » et « vérité du monde », comme en un parfait miroir avec la double modalité de la manifestation théorisée par la phénoménologie radicale. Selon Henry, il s'agit d'une différence phénoménologique décisive. Tandis que la vérité du monde se fonde sur une phénoménalité où subsiste la distinction entre ce qui se manifeste et le «se manifester », la vérité du christianisme implique au contraire une « signification phénoménologique pure » où l'apparaître et ce qui apparaît ne font qu'un ; c'est pourquoi « ce qui se révèle, c'est la révélation elle-même, une révélation de la révélation, une auto-révélation dans sa fulguration originelle immédiate ». Et c'est justement ce que signifie l'affirmation selon laquelle «Dieu est cette Révélation pure qui ne révèle rien d'autre que soi $»{ }^{4}$ Dieu donc se révèle, tout d'abord en se révélant à soi ; en ce sens l'autorévélation de Dieu n'est que le Logos qui, comme dit le Prologue de Jean, « était chez Dieu, était Dieu ».

Or, qu'est-ce qui à proprement parler se révèle par l'autorévélation de Dieu ? Que Dieu est Vie. Dieu se révèle comme vie et dans la vie : «Dieu est Vie, [...] l'essence

\footnotetext{
${ }^{1}$ Ibid., p. 128-129

${ }^{2}$ Ibid., p. 131.

${ }^{3}$ Ibid., p. 132.

${ }^{4}$ M. Henry, C'est moi la vérité. Pour une phénoménologie du christianisme, Paris, Seuil, 1996, p. 36-37.
} 
de la Vie, ou, si l'on préfère, l'essence de la Vie est Dieu », tel est ce qui constitue la «première équation fondamentale du christianisme. ${ }^{1}$ On saisit alors la connexion entre la vérité et la vie dans ce que le Christ dit de lui-même, et c'est pourquoi la notion de vie devient la clef de voûte de l'interprétation henryenne du christianisme. Or qu'est ce que la vie chez Henry? Nous le savons : ce qui n'apparaît pas et pourtant se manifeste, non dans le dehors du monde, car dans ce dehors nous voyons seulement des vivants, et non pas la vie. Plus exactement, nous voyons des hommes, car ce qui fait d'eux les vivants qu'ils sont échappe à la possibilité du regard. La vie se laisse percer uniquement de l'intérieur, par l'expérience de l'autoaffection qui transit l'existence de fond en comble. Si c'est le propre de la vie de se révéler, d'être révélation absolue, comment parvient-elle à se révéler ? Comme Logos, dit Jean, et avec lui toute la tradition chrétienne. Non en un sens purement linguistique toutefois — «logocratique », dirait George Steiner —, car comme le souligne Henry, son action est celle d'engendrer, de donner la vie; vie engendrée dans l'autoengendrement même de la vie, car une même vie étreint Dieu et son Verbe.

Nous touchons ici au deuxième aspect fondamental de la révélation de Dieu, c'està-dire à ceci que « le Logos, le Verbe s'est fait chair» (Jean 1, 14). Ce qui signifie que le Verbe n'a pas reçu simplement un corps, ne s'est pas revêtu d'une matière à lui étrangère, comme il arrivait de temps en temps aux dieux olympiens. Bien au contraire, il s'est fait chair, chair vivante de bout en bout. C'est pourquoi le Christ n'est en rien le frère d'Hercule et de Dionysos, comme le voulait Hölderlin. Selon Henry, il s'agit finalement du «pas le plus difficile » à accomplir pour entendre la parole propre du christianisme : son Logos «n'est pas un Logos qui, perdu dans la contemplation des archétypes intelligibles, doit se revêtir d'un corps matériel semblable à celui des choses; c'est le Logos de la vie, celui de la première lettre de Jean ». ${ }^{2}$ Et on lit dans Incarnation : «Le corps matériel et mondain des Grecs est semblable à ce morceau de terre qui devient chair sous le souffle divin — qui est le souffle de la Vie. Seulement, lorsque le corps est transformé en chair par l'opération de la Vie, il ne tient sa condition de chair que de la Vie ${ }^{3}{ }^{3}$

Il faut donc souligner que l'incarnation chez Henry n'a rien à voir avec la Menschwerdung Gottes, la kénose du Verbe de la pensée idéaliste, en particulier hégélienne, de l'Infini qui se fait fini, de l'irruption de l'éternité dans le temps. En cette vision où l'esprit se fait autre que soi pour revenir à soi, l'incarnation est affirmée pour être niée, car l'esprit comme la phénix renaît toujours de ses cendres. Chez Henry, l'incarnation n'indique pas du tout le moment de la négativité nécessaire à la

\footnotetext{
${ }^{1}$ Ibid., p. 40.

${ }^{2}$ M. Henry, «Eucaristia e fenomenologia nella riflessione filosofica contemporanea », dans N. Reali (éd.), Il mondo del sacramento. Teologia e filosofia a confronto, Edizioni Paoline, Cinisello Balsamo, 2001, p. 129.

${ }^{3}$ M. Henry, Incarnation. Une philosophie de la chair, Paris, Seuil, 2000, p. 191.
} 
positivité, car la phénoménologie de l'immanence se refuse à toute vision dialectique du réel. Il en découle une affirmation fondamentale, tautologique seulement en apparence : « La chair est justement la façon dont la vie se fait Vie. Pas de Vie sans une chair, mais pas de chair sans Vie ». ${ }^{1}$ Partant, « l'abyssale affirmation de Jean », — «Et le Verbe s'est fait chair» —, entendue en toute sa radicalité, ne peut que signifier que « la chair du Verbe ne provient pas du limon de la terre, mais du Verbe lui-même », car "dans le limon de la terre, il n'y a que des corps, aucune chair. Quelque chose comme une chair ne peut advenir et ne nous advient que du Verbe ». ${ }^{2}$

Un autre grand penseur du $\mathrm{XX}^{\mathrm{e}}$ siècle a établi un rapport aussi étroit entre incarnation et langage - je pense à Gadamer. Dans des passages très prégnants de Vérité et méthode, il en vient à affirmer qu'on ne peut percer la vraie essence du langage qu'à partir de l'incarnation chrétienne : «Il y a cependant une pensée qui n'est pas grecque et qui rend mieux justice à l'être de la langue [...]. C'est la pensée chrétienne de l'Incarnation. L'incarnation n'est évidemment pas in-corporation ». Et un peu plus loin : «L'Incarnation est en relation étroite avec le problème de la parole ». Or voici l'affirmation décisive : «Quand la Parole se fait chair et que la réalité de l'esprit ne s'accomplit que dans cette Incarnation, le logos est libéré de sa spiritualité, qui signifie en même temps sa potentialité cosmique ». Il s'ensuit que, «à la différence du Logos grec, le Verbe est pur événement $\gg{ }^{3}$ Tout proches que soient sur ce point les deux philosophes, ce qui les sépare est cependant évident. Chez Gadamer en effet, l'incarnation constitue essentiellement le medium de l'historicité de l'esprit, source du langage ; ainsi, plus qu'à l'essence pathétique de l'incarnation, c'est à la vision hégélienne de la kénose du Logos, de la Menschwerdung Gottes, qu'il fait ici référence. En d'autres termes, la méditation de l'incarnation chrétienne vise chez Gadamer essentiellement la Sprachlichkeit de l'expérience herméneutique, son essence linguistique, tandis que, chez Henry, il en va surtout de sa phénoménologie de la vie comme immanence et affectivité. C'est pourquoi la vérité du christianisme ne peut chez celui-ci être confiée à l'exégèse des textes, car une telle notion de vérité se refuse à toute vérification d'ordre objectif. C'est justement le thème de Paroles $d u$ Christ, où vient en question le problème du langage dans son rapport avec la vie que le Christ a promise aux hommes. En effet, comme le dit Henry, il s'agit de paroles d'un homme qui n'est pas seulement un homme mais le Fils de Dieu, qui parle aux hommes, dans la langue des hommes, pour dire des choses qui ne sont pas seulement humaines - des paroles, surtout, tout à fait différentes de ce que les hommes considèrent être la réalité. D'où la duplicité de la parole du Christ, de la même manière qu'est «double » sa nature. En tant que double, la vérité du christianisme ne peut qu'être paradoxale, comme est paradoxal et invraisemblable le

\footnotetext{
${ }^{1}$ Ibid., p. 174.

${ }^{2}$ Ibid., p. 27.

${ }^{3}$ H-G. Gadamer, Vérité et Méthode, Paris, Seuil, 1996, p. 441-442.
} 
langage qui l'exprime. Voilà la réponse, l'unique possible, à l'objection formulée par J-L. Chrétien au sujet de la difficulté, voire de l'impossibilité de traduire une langue dans l'autre en raison de leur hétérogénéité absolue. Que les deux paroles doivent pouvoir se traduire l'une dans l'autre, si l'on ne veut pas tomber dans la « schizophrénie spirituelle », c'est du reste ce qu'il reconnaît lui-même. ${ }^{1}$ Quant à lui, Michel Henry l'affirme en soulignant : "La parole des hommes ne se réduit nullement à une parole du monde, c'est d'abord celle de la vie » ${ }^{2}$; d'où leur "affinité décisive », quoique paradoxale.

Ainsi l'opposition entre vérité du monde et vérité de la vie est-elle la même que celle qui subsiste entre visible et invisible, où l'invisible est un avec l'immanence de la vie et l'affectivité qui la constitue. C'est pourquoi « la Parole de la Vie parle, non des choses ou du monde, mais de la Vie elle-même. Ou, pour mieux dire, elle parle la vie, ce qu'elle dit, elle ne le pose jamais hors d'elle, mais elle le garde en elle comme un bien dont elle ne se défait jamais, puisqu'il est sa propre vie ». ${ }^{3}$

\footnotetext{
${ }^{1}$ Art. cit., p. 160.

${ }^{2}$ M. Henry, Paroles du Christ, Paris, Seuil, 2002, p. 129.

${ }^{3}$ Ibid., p. 132.
} 
